\title{
Research on Vibration Characteristics of Gun Rotating Parts
}

\author{
Pengfei Yue ${ }^{1}$, Yongyong Zhu ${ }^{1}$, Yang Zhao ${ }^{1}$, Deshi Wang ${ }^{1}$ \\ ${ }^{1}$ Naval University of Engineering, Wuhan 430033, China;
}

\begin{abstract}
Artillery backlash angle is an important factor in firing accuracy. It is necessary to study the law of the change of the backlash angle in order to master the dynamic error of artillery rotating parts in the high and low direction. The vibration equation of backlash angle was deduced based on the force analysis of the rotating parts. The structure and physical parameters of artillery were selected, and the influence of different factors on the backlash angle was analyzed. The case analysis shows that the torsional rigidity of the high and low machine should be designed within an appropriate range according to the natural frequency of the vibration. The structure parameters of the gun have important influence on the vibration characteristics of the rotating parts. In the design of the gun, the center of the trunnion and the center of gravity should be located on the same side of the barrel axis. The research work provides a reference for the further study of the control of the backlash angle.
\end{abstract}

\section{Introduction}

Each part of the gun's rotating parts has a certain inertia, and rotating part constitutes an inertial system as a whole; the shaft system that transmits pitching and gyration motions in the gun is elastic, and even the gap in the transmission system will cause a backlash; the trunnion connection part itself contains a damping factor, and the friction between the components also causes the damping effect. In this way, the rotating part has the three elements of vibration inertia, stiffness, and damping, and becomes a vibration system. When shooting, the impact force generated by the internal ballistic process and the motion excitation force of the projectile provide initial energy for the vibration of the system, so that the rotating parts of the artillery and its components will be in a state of vibration. Under vibration conditions, it is difficult to eliminate the empty loop angle generated by the rotation of the landing part around the trunnion, which has an important impact on shooting accuracy. When the artillery is fired, the force on the landing part is complicated. Орлов established the dynamic equation of the air-return angle during the interior ballistic period.
Based on the classic gun body structure and design theory, and on the basis of the analysis steps of the article, combined with the law of recoil and reclamation of the artillery, a dynamic model of the rotating part of the shooting process is established, and the empty return angle equation is derived.

\section{Basic assumptions and physical models}

The definition of the blacklash angle: The angle between the chord line at the center of the cross-line at both ends of the upper body tube at the resting position and the chord line at the center of the cross-line center at both ends of the body tube when the projectile flies out of the muzzle, and the latter line is the ideal centerline of the barrel without considering the elastic deformation of the barrel and manufacturing errors. When calculating the air return angle, only the high and low gear arc machine and other artillery parts are driven by the impact to drive the rising and falling parts to rotate around the trunnion. In order to study the vibration of the rotating parts of the artillery, consider the force and motion state of the landing part, as shown in figure 1 . 


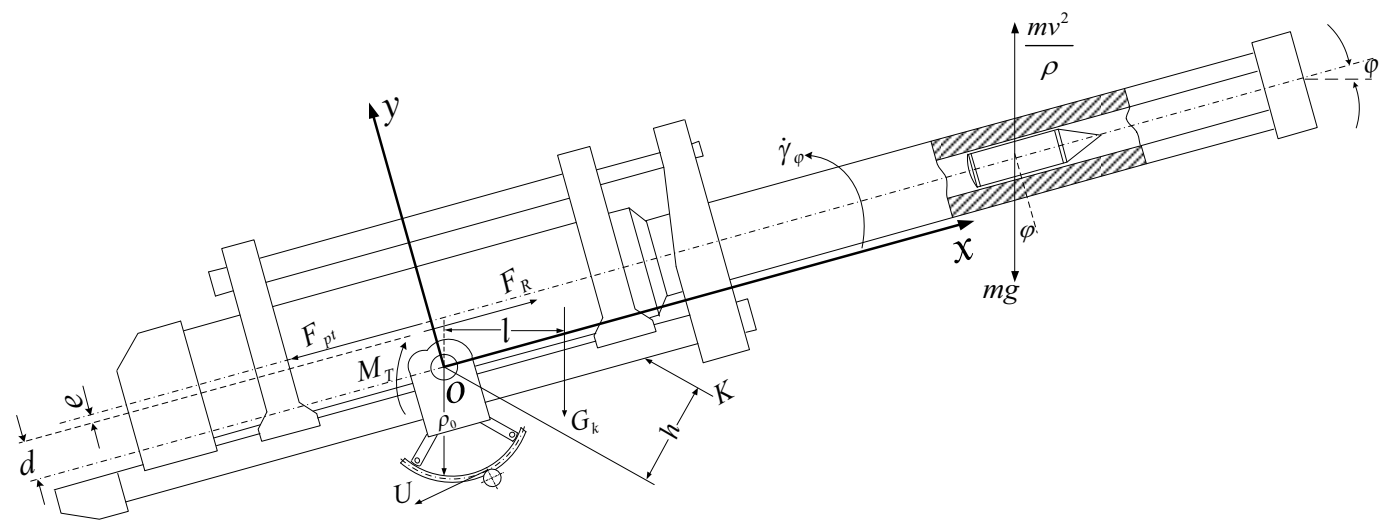

Figure.1. Force diagram for rotating parts of gun

Assumption: (1) The rotating parts of the artillery is an absolute rigid body. In this case, the rising and rotating parts are inelastic, the curvature radius $\rho$ of the barrel can be regarded as a constant; (2) The trunnion does not move during internal ballistics, that is, the trunnion is regarded as a fixed axis, and the recoil part and the body tube rotate around the trunnion as a whole; (3) When launching, all external forces and external moments on the rotating parts act on the launch surface.

Under the assumption (3), the force on the rotating parts can be simplified to a plane force system. Take the trunnion as the coordinate origin $o$, and establish a plane rectangular coordinate system $o x y$, in which the $x$-axis is parallel to the axis of the barrel, the direction of the muzzle is positive, and the $y$-axis is perpendicular to the $x$-axis. Based on the above-mentioned basic assumptions and the physical model of the ups and downs, dynamic equations can be established, and the change law of the backlash angle can be further analysed. Note that $m$ is the mass of the projectile, $v$ is the movement speed of the projectile, and $P_{d}$ is the bottom pressure of the projectile; $M$ is the mass of the recoil part, $V$ is the movement speed of the recoil part, $F_{R}$ is the resultant force of the recoil resistance, $F_{p t}$ is the resultant force of the barrel, and the directions of $F_{R}$ and $F_{p t}$ are parallel to the direction of the bore axis, and $F_{R}$ acts on the center of gravity of the recoil. $\varphi$ is the angle of fire, $\gamma_{\varphi}$ is the angular velocity of the empty return, $G_{k}$ is the gravity of the landing and landing part, $l$ is the force arm of the gravity of the rotating parts against the center of the trunnion, and $K$ is the pushing (or pulling) force provided by the balancer to the landing and landing part, $h$ is the force arm of the balancer spring against the center of the trunnion, $U$ is the force on the high and low tooth arcs of the cradle, $\rho_{0}$ is the force arm of the force $U$ against the center of the trunnion, and $M_{T}$ is the friction moment on the trunnion. The bore axis is an arc with a large radius of curvature, which represents the centrifugal force of the projectile curve movement. Considering that the radius of curvature is much larger than the barrel length, the muzzle angle of large and medium caliber guns is generally negative, and the direction of centrifugal force is vertically upward.

\section{Kinetic equation of the gun rotating part}

During the interior ballistic period, the motion equation of the projectile and recoil is

$$
\begin{gathered}
\varphi_{1} m \frac{d v}{d t}=P_{d} \\
M \frac{d V}{\mathrm{~d} t}=F_{p t}-F_{R}
\end{gathered}
$$

In the formula, $\varphi_{1}$ is the calculation coefficient of the secondary work. Known from interior ballistics, $P_{d} / F_{p t}=\varphi_{1} /\left(1+\frac{\omega}{2 m}\right)$, which $\omega$ is the gunpowder charge. The momentum moment $L_{o k}$ of the rotating part to the trunnion center is composed of the momentum moment $L_{o h}$ of the recoil part to the trunnion center, the momentum moment $L_{o c}$ of the projectile to the trunnion center, and the momentum moment $L_{o p}$ of the cradle to the trunnion center, namely

$$
L_{o k}=L_{o h}+L_{o c}+L_{o p}
$$

Where, the momentum moment $L_{o p}$ of the cradle to the trunnion center is expressed as

$$
L_{o p}=J_{p} \dot{\gamma}_{\varphi}
$$

In the formula, $J_{p}$ is the moment of inertia of the cradle to the center of the trunnion.

Regarding the projectile as a rigid body moving in $m_{i}$ plane, a represents the mass of the projectile's microelement body, and $\boldsymbol{r}_{i}$ represents the vector diameter from the center of the trunnion to the micro-element body, then the projectile momentum $L_{o c}$ can be expressed as

$$
L_{o c}=-\dot{\gamma}_{\varphi} \sum m_{i}\left(x_{i}^{2}+y_{i}^{2}\right)-m v(d+e)
$$

Where $x_{i}^{2}+y_{i}^{2}=r_{i}^{2}, d$ represents the distance from the center of gravity of the rear seat to the center line of the trunnion, and $e$ represents the distance between 
the bore axis and the center of gravity of the rear seat. According to the right-hand screw rule, it can be determined that the momentum $L_{o c}$ is clockwise, so take the minus sign. Similarly, the moment of momentum $L_{o h}$ of the recoil part can be expressed as

$$
L_{o h}=\dot{\gamma}_{\varphi} \sum M_{i}\left(X_{i}^{2}+Y_{i}^{2}\right)+M V d
$$

On the basis of the above, according to the theorem of moment of momentum, establish the dynamic equation of the gun rotating parts.

$$
\begin{aligned}
& {\left[J_{p}+\sum M_{i}\left(X_{i}^{2}+Y_{i}^{2}\right)-\sum m_{i}\left(x_{i}^{2}+y_{i}^{2}\right)\right] \ddot{y}_{\varphi}+2(M V X-m v x) \dot{\gamma}_{\varphi}} \\
& =F_{p l}\left[(d+e) /\left(1+\frac{\omega}{2 m}\right)-d\right]+F_{R} d+K h-Q_{k} l-U \rho_{0}-m g l_{x}+m v^{2} l_{x} / \rho-M_{T}
\end{aligned}
$$

Where $l_{x}$ is the force arm of the projectile's gravity.

$$
\left[J_{p}+\sum M_{i}\left(X_{i}^{2}+Y_{i}^{2}\right)\right] \ddot{\gamma}_{\varphi}+2 M V X \dot{\gamma}_{\varphi}=\left(F_{R}-F_{p t}\right) d+K h-Q_{k} l-U \rho_{0}-M_{T}
$$

After the end of the interior ballistic period, the impact of the projectile disappears, and the dynamic equation of the rotating part is

$$
\left[J_{p}+\sum M_{i}\left(X_{i}^{2}+Y_{i}^{2}\right)\right] \ddot{\gamma}_{\varphi}+2 M V X \dot{\gamma}_{\varphi}=Q_{k} l-K h-F_{r} d-U \rho_{0}-M_{T}
$$

Where $F_{r}$ represents the resultant force of recuperation. According to the design principle of the balancing machine, there are the following relations when sitting back:

$$
K h-Q_{k} l=\Delta M+M g X \cos \varphi
$$

In the above formula, $X$ represents the movement distance of the recoil part, and $\Delta M$ is the unbalanced moment. During the internal ballistic period, $\Delta M$ fluctuates slightly and does not change direction. The reaction torque of the gear teeth on the high and low crankshaft can be expressed as the product of the empty return angle and the torsional stiffness $k$.

$$
\begin{gathered}
U \rho_{0}=k \gamma_{\varphi} \\
\gamma_{\varphi}(t)=\frac{\omega_{n} \zeta \gamma_{\varphi 0}+\dot{\gamma}_{\varphi 0}}{\omega_{d}} e^{-\omega_{n} \zeta t} \sin \left(\omega_{d} t\right)+\gamma_{\varphi 0} e
\end{gathered}
$$

Where $\omega_{d}$ is the damped vibration frequency, $\omega_{d}=\omega_{n} \sqrt{1-\zeta^{2}}$.

\section{The influence of different parameters on the vibration of the rotating part}

The resultant force of the gun bore is the main force on the rise and fall of the interior ballistic period, which can be obtained from the difference of experimental data and fitting. In the period of internal ballistics, the 5th-order polynomial model is used to obtain the expression of the resultant barrel force

$$
F_{p t}=\left[a_{0}+a_{1} t+a_{2} t^{2}+a_{3} t^{3}+a_{4} t^{4}+a_{5} t^{5}\right] \mathrm{A}
$$

In the above formula, $\mathrm{A}$ is the cross-sectional area of the barrel. In the after-effect period, the empirical formula is generally used to calculate the resultant force of the barrel.

$$
F_{p t}=F_{p t g} e^{-\frac{t-t_{g}}{b}} \quad\left(t>t_{g}\right)
$$

The friction moment $M_{T}$ of the trunnion of the cradle can be expressed as the product of the empty return angular velocity and the damping coefficient $c$.

$$
M_{T}=c \dot{\gamma}_{\varphi}
$$

\section{Solution of backlash}

Taking the dynamic equations of the up and down part of the interior ballistic period as an example, the method of solving the recoil and recoil period is the same as that of the interior ballistic period, and we can get

$$
\begin{aligned}
& {\left[J_{p}+\sum M_{i}\left(X_{i}^{2}+Y_{i}^{2}\right)-\sum m_{i}\left(x_{i}^{2}+y_{i}^{2}\right)\right] \ddot{\gamma}_{\varphi}+[2(M V X-m v x)+c] \dot{\gamma}_{\varphi}+k \gamma_{\varphi}} \\
& =F_{p t}\left[(d+e) /\left(1+\frac{\omega}{2 m}\right)-d\right]+F_{R} d+\Delta M+M g X \cos \varphi+m v^{2} l_{x} / \rho-m g l_{x}
\end{aligned}
$$

Let $J_{k}$ as the moment of inertia of the landing part relative to the center of the trunnion, then

$$
J_{k}=J_{p}+\sum M_{i}\left(X_{i}^{2}+Y_{i}^{2}\right)-\sum m_{i}\left(x_{i}^{2}+y_{i}^{2}\right)
$$

Let $M(t)$ as the instantaneous value of the resultant moment, then

$$
M(t)=F_{p t}\left[(d+e) /\left(1+\frac{\omega}{2 m}\right)-d\right]+F_{R} d+\Delta M+M g X \cos \varphi+m v^{2} l_{x} / \rho-m g l_{x}
$$

Let the natural frequency $\omega_{n}$ and damping ratio $\zeta$ of air-return motion be

$$
\begin{gathered}
\omega_{n}=\sqrt{k / J_{k}} \\
\zeta=\frac{2(M V X-m v x)+c}{2 \sqrt{k \cdot J_{k}}}
\end{gathered}
$$

The dynamic equation of the rotating parts can be further simplified as

$$
\ddot{\gamma}_{\varphi}+2 \omega_{n} \zeta \dot{\gamma}_{\varphi}+\omega_{n}^{2} \gamma_{\varphi}=M(t) / J_{k}
$$

According to Laplace transform, we can get

$$
\cos \left(\omega_{d} t\right)+\frac{1}{\omega_{d} J_{k}} \int_{0}^{t}\left[M(\tau) e^{-\omega_{n} \zeta(t-\tau)} \sin \left(\omega_{d}(t-\tau)\right)\right] d \tau
$$

Where $t_{g} \mathrm{t}$ is the internal ballistic time, $F_{p t g}$ is the resultant bore force at the end of the interior ballistic, and $b$ is the attenuation coefficient of the resultant bore force. The treatment of recoil resistance and recoil force is similar to the resultant force of the barrel, and both can be obtained by fitting the difference of experimental data. $a_{0}=5.55 \times 10^{7}, \quad a_{1}=-8.56 \times 10^{10}, a_{2}=1.68 \times 10^{14}$, $a_{3}=-5.54 \times 10^{16}, a_{4}=6.79 \times 10^{18}, a_{5}=-2.88 \times 10^{20}$. Other input parameters are shown in Table 1.

Table.1 Input parameters of simulation

\begin{tabular}{cc|cc}
\hline Parameter & Value & Parameter & Value \\
\hline$m / \mathrm{kg}$ & 15.6 & $A / \mathrm{m}^{2}$ & 0.00785 \\
$M / \mathrm{kg}$ & 1550 & $J_{k 0} / \mathrm{kg} \cdot \mathrm{m}^{2}$ & 2155 \\
$\omega / \mathrm{kg}$ & 5.5 & $\rho / \mathrm{m}$ & 1000 \\
$\mathrm{k} / \mathrm{N} \cdot \mathrm{m} \cdot \mathrm{rad}^{-1}$ & $1.8 \times 10^{7}$ & $\varphi / \mathrm{rad}$ & $\pi / 4$ \\
$e / \mathrm{m}$ & 0.03 & $\varphi_{1}$ & 1.02 \\
$d / \mathrm{m}$ & 0.03 & $b$ & 0.01 \\
\hline
\end{tabular}


Change the torsional stiffness of the high and low machine to $1.6 \times 107 \mathrm{~N} \cdot \mathrm{m} / \mathrm{rad}, \quad 1.8 \times 107 \mathrm{~N} \cdot \mathrm{m} / \mathrm{rad}$,

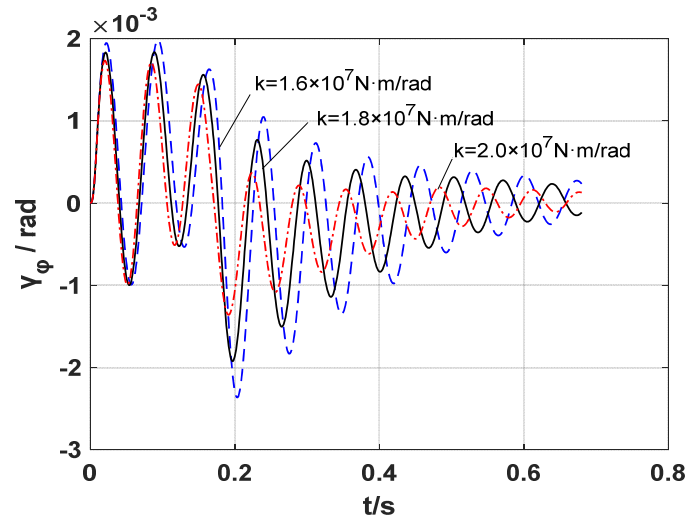

Figure 2. Relationship between backlash angle and elevating mechanism rigidity.

As shown in Figure 2, as the torsional stiffness of the high-low machine increases, the amplitude of the empty return angle decreases and the frequency of vibration increases. The negative direction empty return angle value is more obviously affected by the high-low machine torsional stiffness. As shown in Figure 3, the oscillation trend of the empty loop angle decreases with the increase of the damping ratio. However, the torsional stiffness of the high-low machine cannot be reduced indefinitely,

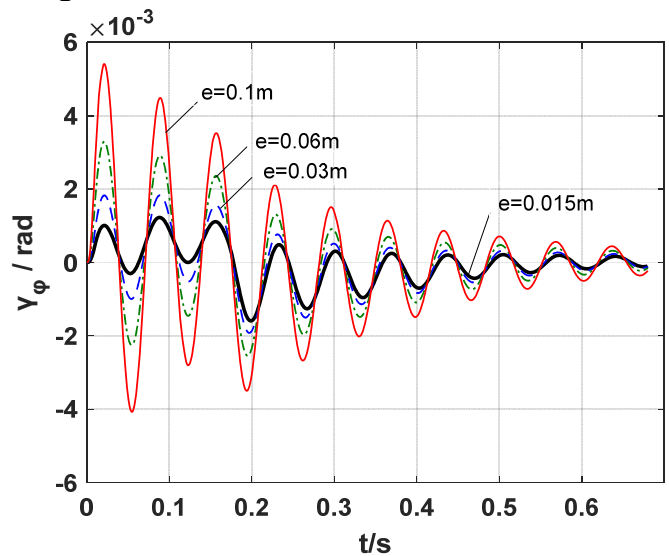

Figure 4. Relationship between backlash angle and $\mathrm{e}$

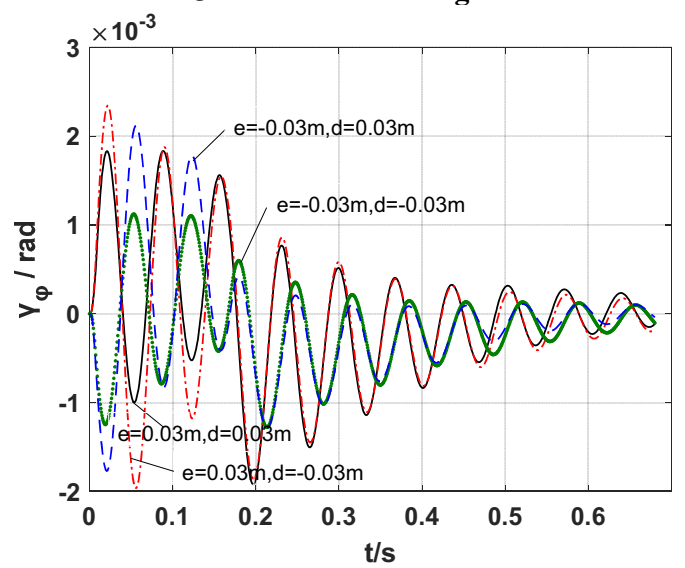

Figure 6. Relationship between backlash angle and positive or negative e and d

In the structural parameters of the artillery, the distance between the barrel axis and the center of gravity of the recoil is expressed as e, and the distance from the center
$2.0 \times 107 \mathrm{~N} \cdot \mathrm{m} / \mathrm{rad}$, and other initial conditions remain unchanged, and carry out numerical simulation, the results are shown in Figures 2 and 3.

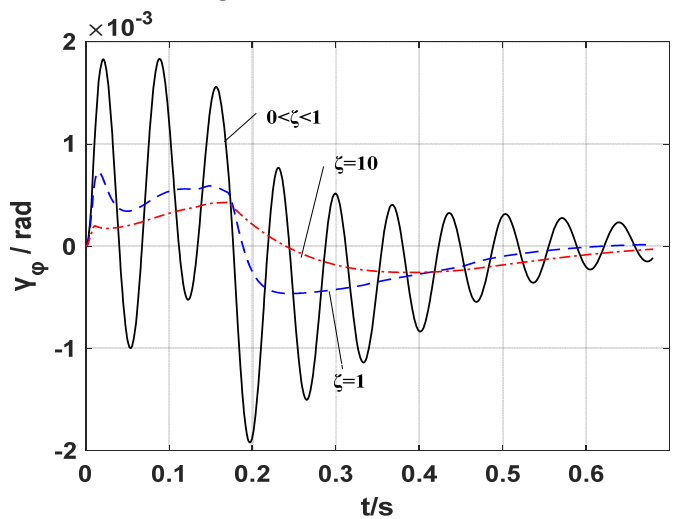

Figure 3. Relationship between backlash angle and relative damping factor

because the decrease of the stiffness increases the empty return angle value. Therefore, in order to control the empty return angle and reduce the oscillation of the system, on the one hand, it is necessary to select the appropriate rigidity of a certain flexible component, such as a flexible coupling with appropriate rigidity; On the other hand, additional components that provide damping can be added, such as designing brakes for high and low crankshafts to ensure the irreversibility of transmission.

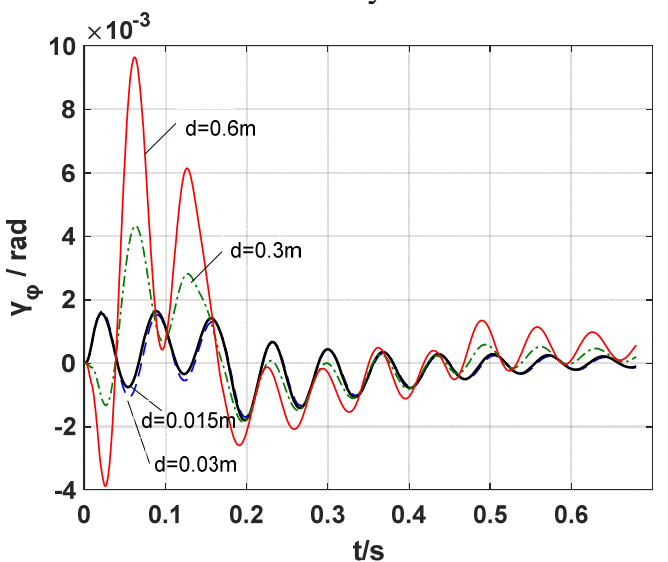

Figure 5. Relationship between backlash angle and d 
vibration response of the system, and it is necessary to analyze them. When $\mathrm{d}$ is $0.03 \mathrm{~m}$, e is $0.015 \mathrm{~m}, 0.03 \mathrm{~m}$, $0.06 \mathrm{~m}, 0.1 \mathrm{~m}$; when e is $0.03 \mathrm{~m}$, $\mathrm{d}$ is $0.015 \mathrm{~m}, 0.03 \mathrm{~m}, 0.3 \mathrm{~m}$, $0.6 \mathrm{~m}$, Study the influence of structural parameters e and $\mathrm{d}$ on the empty backlash of the rotating section, The results are shown in Figure 4 6.

According to the parameters selected in this paper, as shown in Figure 4, as e increases, the amplitude of the empty loop angle increases; as shown in Figure 5, as d increases, the amplitude of the empty loop angle increases, When the value of $d$ increases to a certain degree, the direction of system vibration is reversed. As shown in Figure 6, the values of e and $\mathrm{d}$ are unchanged but their signs are changed. The positional relationship between the barrel axis, the center of gravity of the recoil and the center of the trunnion has a greater impact on the vibration law of the system. When the barrel axis is on the center of gravity of the recoil, the initial response of the system vibration is counterclockwise. If the centerline of the trunnion is arranged on the center of gravity of the recoil, the maximum amplitude of the empty loop angle is greater. When the barrel axis is below the center of gravity of the recoil, the initial response direction of the system vibration is clockwise. If the centerline of the trunnion is arranged below the center of gravity of the recoil, the maximum amplitude of the backlash is greater. In summary, when the signs of $\mathrm{e}$ and $\mathrm{d}$ are the same, the maximum amplitude of the air-return angle vibration is smaller, and the simulation results meet the requirements of controlling the vibration of the landing part in the gun mount design theory. Therefore, in the design, it is best to make the center of the trunnion and the center of gravity of the recoil part on the same side of the bore axis.

\section{Conclusion}

In this paper, a dynamic model of the gun's rise and fall during the shooting process is established, and the airreturn angle vibration equation is derived. Through numerical simulation, the influence of different parameters on the backlash is studied. The conclusions are as follows:

(1) Increasing the torsional stiffness of the high-low machine is conducive to reducing the amplitude of the backlash, but the vibration frequency of the rotating part increases, and the low-amplitude and high-frequency motion components at the muzzle increase. To determine the range of torsional stiffness of the high and low crankshaft, the requirements of the natural frequency of the whole gun vibration and the natural frequency of the air-return vibration of the gun rotating parts should be considered comprehensively.

(2) During the design of the artillery structure, the distance between the center of gravity of the recoil and the axis of the bore and the distance between the center of gravity of the recoil and the center of the trunnion should be minimized. From the perspective of controlling the vibration of the rotating part, the center of the trunnion and the center of gravity of the recoil part should be located on the same side of the bore axis.

\section{References}

1. Wang Deshi. Theory on vibration of gun[M].Beijing: Weapon Industry Press, 2015:12-29.

2. Mi Liangchuan, Shi Yubin, Wang Pengfei. Influence of barrel straightness error on projectile jump disturbance[J]. Journal of ballistics,2013,25(1): 94-98

3. Mi Liangchuan, Liu Min, Gao Shuzi. Mathematic description and test analysis on launched projectile muzzle velocity jumping angle during howitzer firing $[\mathrm{J}]$. Journal of gun launch and control,2013,3:13.

4. Peng Zhiguo, Zhou Yanhuang, Qi Liting. Analysis of First round deviation factors and treatment of experiment data[J]. Journal of gun launch and control, 2007,12(4): 1-5.

5. Б. В. Орлов, Э. К. Ларман, В. Г. Маликов. Structure and design for barrel [M]. WANG Tianhuai, LIU Shuhua, translated. Beijing: National Defense Industry Press, 1982:169-187.

6. PU Fa.The formation ang estimation of the muzzle angle ang its effect upon the jump angle and firing[J].ACTA Armamentar[J],1983,4(1): 1-8.

7. JIN Zhiming. Gun interior ballistics[M]. Beijing: Beijing Institute of Technology Press,2004,63-66.

8. Zhang Xunguo. Research on optimization design of the screw gas pressure elevating and equilibrating mechanism[M]. Nan Jing: Nanjing University of Science and Technology.

9. ZHOU Dekui,WANG Ruxin, YING Yanlin. Analysis of equilibrator for naval gun[J]. Journal of Naval Academy of Engineering, 1993(2): 56-62.

10. Shi Yuedong, Wang Deshi. Analysis of naval vibration based on Guass minimum constraint theory[J]. Journal of Naval University of Engineering, 2009(10): 1-5. 